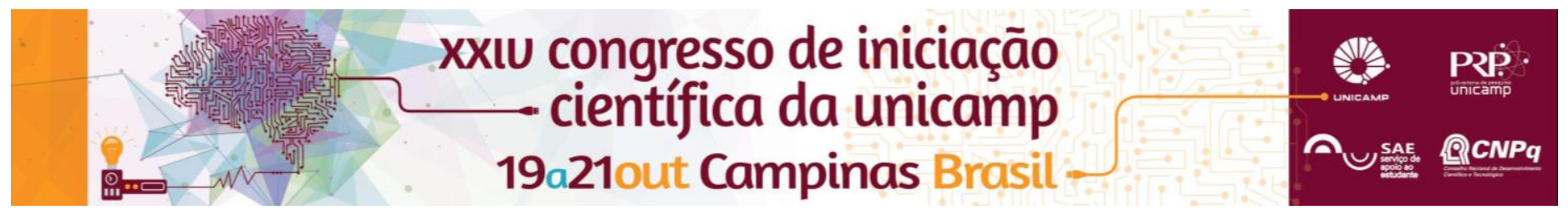

\title{
Automated system for Nuclear Magnetic Resonance probes
}

\author{
Luiz H. M. Marchesini ${ }^{*}$ Alim Gonçalves, Ricardo R. Urbano.
}

\begin{abstract}
In this multidisciplinary work plan we developed an automated system able to control the tuning of the resonant frequency of a tank (RLC) circuit in Nuclear Magnetic Resonance (NMR) probes. Thus, the variable capacitors - tuning (CT) and matching (CM) - of such a circuit are remotely controlled with the aid of engines (DC motor) via a computer interface quite quickly and very conveniently from the desk. This technical improvement certainly makes a great difference in NMR experiments in the research of complex and advanced materials in Condensed Matter Physics developed at the IFGW-Unicamp.
\end{abstract}

Key words: Automation, nuclear magnetic resonance, computer interface.

\section{Introduction}

Nuclear Magnetic Resonance Spectroscopy (NMR) is a technique that exploits the magnetic properties of certain atomic nuclei. It was first described and measured in molecular beams and later expanded for use in liquids and solids.

In order to carry out solid-state NMR in magnetic materials, most of time is necessary to sweep the frequency within a wide range $(\Delta v \sim 1$ to $5 \mathrm{MHz})$ accumulating the signal obtained point-to-point. The final broad NMR spectrum is then reconstituted by combining all these steps together through a mathematical procedure named FFT-Sum.

Therefore, the automated system able to remotely control the tuning of the resonant frequency of a tank circuit in NMR probes proposed in this project is of utmost importance during NMR experiments. This allows adjustment of the tank circuit resonant frequency to coincide with the Larmor frequency of the nucleous under study in a very fast and convenient way as it eliminates the need of interrupting a measurement for cable connection/re-connection onto the Network Analyzer often used during a manual frequency tuning.

\section{Results and Discussion}

A tuning box containing the tank (LC) circuit with two variable glass capacitors (Voltronics) and a solenoid with a standard sample was built using an aluminum box and adapters for BNC-connection. This box was used to simulate the circuit inside the NMR probes.

Two DC motors were attached to the variable capacitors and with a circuit $H$ bridge one is able to control the direction of rotation of the engine via a DC-12V circuit using a Arduino Uno board. Also, an RF switch is used to set control either to the NMR Spectrometer or to the Network Analyzer. This summarizes the hardware necessary for the automation of a tank circuit. In other words, this hardware is responsible to do the matching of the resonant frequency of the probed nucleous by controling each engine to the matching (CM) and tuning (CT) capacitors independently.

After several studies and preliminary tests, the interface (computer control code) between the operator and the hardware was created using the LabVIEW program. This interface is shown in Figure 1. Through this interface is possible to switch from Spectrometer (Spec) and Network Analyzer (NA) via an RF Switch as well as to control both DC motors via Arduino Uno board in order to tune the tank circuit to a desired frequency remotely from a computer. The target frequency value and its gain is also shown. There is also a stop button which stops the procedure at any time. Finally, this interface also displays whatever is being shown on the screen of the Network Analyzer for remote access purposes (control the tuning process outside the laboratory).
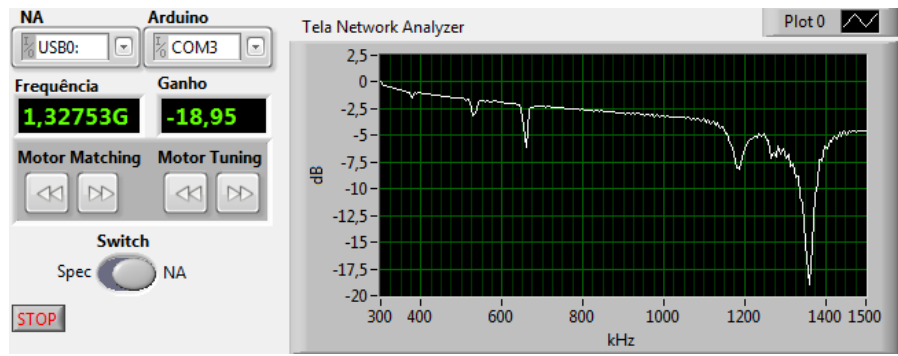

Figure 1. LabVIEW Control Interface of our automated tuning system for solid state NMR experiments.

\section{Conclusions}

An automated tuning system for NMR probes has been developed from scratch. This means that with this system one is capable to control the settings (tuning) of the resonant frequency of a tank circuit in NMR probes via remote access by computer. An operator has full control of the movements of the DC motors through the computer interface and thus is able to properly tune the desired resonant frequency of the tank circuit remotely.

A possible improvement to this system would be developing the autotuning option - an algorithm which is able to seek a target frequency value automatically by running a procedure that looks for the minimum gain value on the Network Analyzer screen and controls the DC motors independently in order to reach a desired frequency value.

\section{Acknowledgement}

We are thankful to the GPOMS and IFGW-UNICAMP staff members for technical assistance. This work was developed under the auspices of FAPESP and CNPq. 